\title{
Vortical Structures in High-Reynolds-Number Jet Indicating Edgetone Oscillation*
}

\author{
Yuta KAWAI**, Yoshiyuki TSUJI*** and Yutaka KUKITA*** \\ ${ }^{* *}$ Aisan Industry Co., Ltd, 1-1-1 Kyowa-cho, Obu, Aichi 474-8588, Japan \\ E-mail: yuta_kawai@aisan-ind.co.jp \\ *** Department of Energy Engineering \& Science, \\ Nagoya University, Nagoya 464-8603, Japan
}

\begin{abstract}
Coherent vortical structures in a high-Reynolds number turbulent jet indicating edgetone oscillations are extracted from velocity and pressure data. Three statistical quantities are adopted as the candidate indicators of coherent structures; (i) phase-averaged vorticity, (ii) phase-averaged pressure, and (iii) phase-averaged velocity gradient tensor. All these quantities work well for extracting coherent structures in a test case of low-Reynolds number cylinder wake. However, when applied to the high-Reynolds number edgetone flow, (ii) and (iii) indicate much better performance than (i), because (i) is affected by the background time-averaged vorticity field. The advection speed of coherent structures is evaluated and compared with Powell's feedback mechanism.
\end{abstract}

Key words: Edgetone, Coherent Structure, Velocity Gradient Tensor, Low Pressure

\section{Introduction}

When a two-dimensional jet collides with a sharp body or an edge, self-sustaining oscillations are induced. This has been called the "edgetone phenomenon", and it is explained by noise feedback due to the vortex sheets impinging on the sharp body ${ }^{(1)}$. As the edgetone is generated by the vortex pairs beside the sharp body, most attempts so far have been made to reduce the noise by intercepting them ${ }^{(2,3)}$. It is generally accepted that the large vortexes are effectively controlled by the initial condition at the nozzle, and then the noise level is dramatically decreased.

Although a large number of studies have been carried out to capture the vortical structures and their impinging, they only covered the low-Reynolds number cases, because the vortical structures could be educed by visualization techniques ${ }^{(4,5)}$. On the other hand, the coherent structures in high-Reynolds number cases have never been sufficiently examined. The flow in a high-Reynolds number edgetone is characterized by periodic velocity and pressure fluctuation, then the data are filtered and phase-averaged to capture the coherent structures in this paper. Three statistical quantities are chosen as candidate indicators of the coherent structures; (i) phase-averaged vorticity, (ii) phase-averaged pressure, and (iii) phase-averaged velocity gradient tensor. First, we ascertain whether all three quantities work well or not for extracting coherent structures in a test case of a low-Reynolds-number wake of a cylinder. Then, they are applied to the high-Reynolds number edgtone phenomenon. In this study, we define the term "vortical structure" as coherent structures with swirling motions, which is identified in the high-Reynolds number edgtone as well as the low-Reynolds number wake of a cylinder. We also examine the feedback loop by estimating the convection speed of vortical structures.

${ }^{*}$ Received 20 Sep., 2007 (No. T1-05-1167) Japanese Original : Trans. Jpn. Soc. Mech. Eng., Vol.72, No.724, B (2006),

pp.3058-3065 (Received 15 Nov., 2005) [DOI: 10.1299/jfst.2.665] 


\section{Experimental Condition}

The edgetone experiment was carried out in an open-circuit wind-tunnel. The aspect ratio of the nozzle is 20 and a two-dimensional jet was made. As indicated in the detailed test section in Fig. 1, edgetone is generated by a two-dimensional jet which collides with the leading edge of the flat plate. The flat plate measures $735 \mathrm{~mm}$ in length, $200 \mathrm{~mm}$ in width, and $6 \mathrm{~mm}$ in thickness. This plate is placed parallel to the flow, and its leading edge is located on the jet center line at $x_{1} / b_{e}=20$ where the jet velocity profile has developed so as to be self preserving. The plate is made of bakelite to decrease the cooling effect by the hot-wire close to the wall. The leading edge is in the shape of an ellipse recommended by Narashimha et al. ${ }^{(23)}$, because it does not generate a separation when the jet flow collides with the plate. We carried out the experiment at $\operatorname{Re} \equiv U_{e} b_{e} / v=22000$ (where $U_{e}$ indicates nozzle exit mean velocity, nozzle exit height $b_{e}=10 \mathrm{~mm}$ and $v$ the kinematic viscosity). Velocity fluctuations are measured by I and X-type probes operated by constant temperature anemometry. Data are sampled by $10 \mathrm{kHz}$ for thirty seconds and digitized by a 12-bit $\mathrm{A} / \mathrm{D}$ converter.
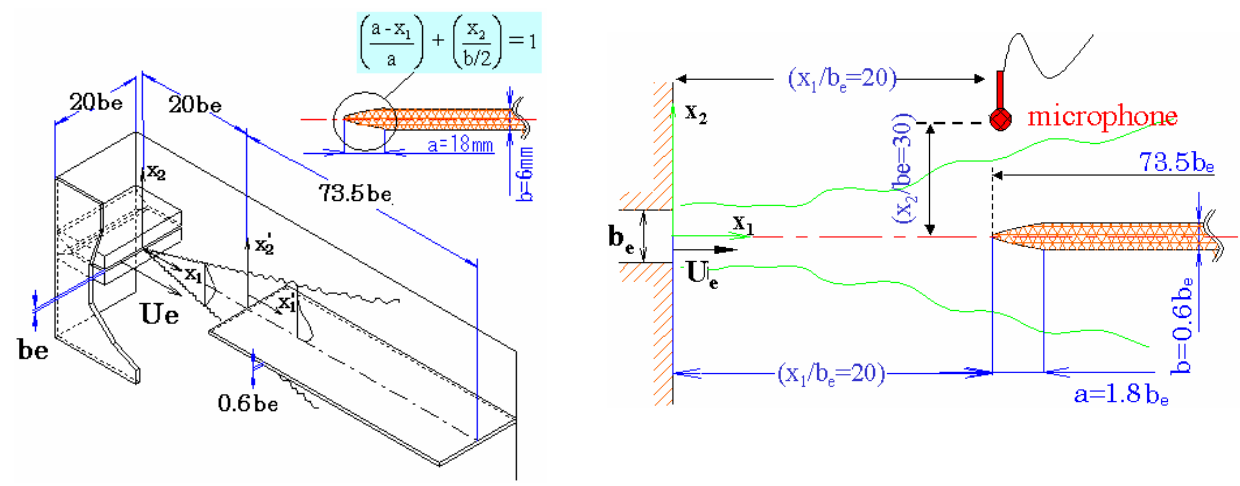

Fig. 1 Schematic view of experimental facility and coordinate system in edgetone experiment.

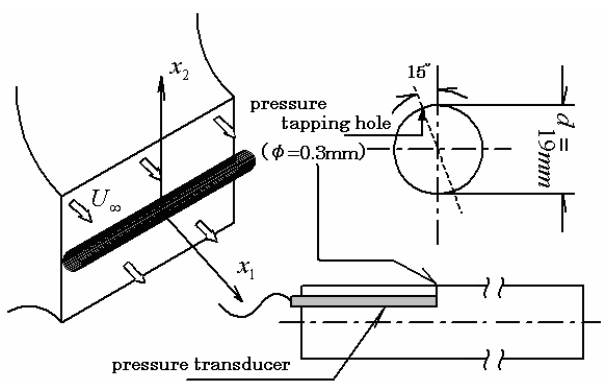

Fig. 2 Schematic view of experimental facility and coordinate system in the wake of circular cylinder.

For a more detailed explanation of the experimental apparatus, see the preceding papers ${ }^{(6,7)}$. In the present study, the flow region in front of the leading edge $(0 \leq x \leq 200)$ is called the "impinging region", and the region on the flat plate is called the "wall region" for convenience.

To conduct experiments on the wake, a cylinder with the diameter $d=19 \mathrm{~mm}$ was attached to the nozzle exit $700 \mathrm{~mm} \times 400 \mathrm{~mm}$ in size. The test section of the wake experiment is shown in Fig. 2. The experimental condition was settled at $\operatorname{Re} \equiv U_{\infty} d / v=3700$ (where $U_{\infty}$ is mean velocity in steady flow upstream of the cylinder). Velocity fluctuations are measured by a X-type probe operated by a constant temperature anemometer. 
The power spectrum obtained by analyzing fluctuating velocity had a pronounced peak in both experiments, which appeared at $40 \mathrm{~Hz}$ in edgetone and $20 \mathrm{~Hz}$ in wake, respectively. We analyzed both flow fields using the conditional sampling method based on each pronounced fluctuating component. In the edgetone experiment, the reference signal for the conditional sampling was the sound pressure received by a microphone fixed at $\left(x_{1}, x_{2}\right)=\left(20 b_{e}, 30 b_{e}\right)$ where no flow influence would reach $^{(7)}$. In the wake experiment, the pressure fluctuation on the cylinder surface was used for the reference signal. Measurements of pressure fluctuation on the cylinder surface were performed with a pressure transducer (KULITE model XCS-062-5G. The eigen frequency of the diaphragm is $150 \mathrm{kHz}$ ) inserted in a cylinder, and the output signals were gained by pre-amplifer. The yaw angle of the pressure holes with $0.3 \mathrm{~mm}$ diameter was 15 degrees (see Fig. 2). In the preliminary experiments, the periodical pressure fluctuations were so pronounced at this yaw angle that the separation point would be around there.

In this study, static pressure fluctuations in the turbulent jet were measured by a static pressure probe to capture voricical structures. The detail of static pressure probe geometry was shown in Fig. 3. The static tube with $1.0 \mathrm{~mm}$ outer diameter and $0.8 \mathrm{~mm}$ bore constructing pressure probe was made of stainless steel. Its 4 pinholes with $0.4 \mathrm{~mm}$ diameter were opened at $22 \mathrm{~mm}$ distance from tip of the static tube, and their center lines crossed each other at right angles. The characteristics of microphone are shown in Table 1. Simultaneous measurements of velocity and pressure fluctuations were carried out with the pinholes at $1.0 \mathrm{~mm}$ from the X-type hot wire.

Table 1 Characteristics of condenser microphone

\begin{tabular}{|c|c|c|c|}
\hline $\begin{array}{c}\text { Sensitivity } \\
(\mathrm{dB} / \mathrm{Pa})\end{array}$ & $\begin{array}{c}\text { diameter } \\
(\text { inch })\end{array}$ & $\begin{array}{c}\text { response } \\
(\mathrm{Hz})\end{array}$ & $\begin{array}{c}\text { Dynamic } \\
\text { range }(\mathrm{dB})\end{array}$ \\
\hline-58 & $1 / 4$ & $20-70 \mathrm{~K}$ & $60-164$ \\
\hline
\end{tabular}

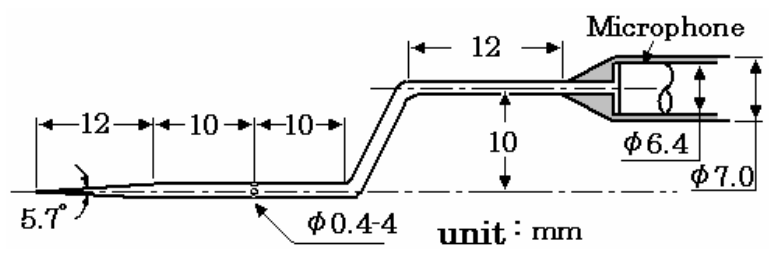

Fig. 3 Schematic of static pressure probe

The static tube with $0.8 \mathrm{~mm}$ bore is much smaller than the microhopne (1/4 inch bore), and there is a small cavity between the static tube and diaphragm of the microphone. These geometries cause the Helmholtz resonance affecting measurements of pressure fluctuations. To eliminate the influence of Helmholtz resonance, at first, we decomposed the sampled pressure fluctuation signal into several frequency bands. Second, amplitude and phase were revised based on the frequency response. Finally, we reconstructed them into a real space. For details on the modification method, see reference (8).

The mean static pressure was measured by a condenser-type pressure sensor (Toyotakouki CS7030A) with a static tube having the same geometry as shown in Fig. 3. The dynamic range of this pressure sensor is $50 \mathrm{~mm} \mathrm{H}_{2} \mathrm{O}$ and its response time is $60 \mathrm{~ms}$.

\section{Nomenclature}

$b_{e}$ : height of nozzle exit $(=10 \mathrm{~mm})$.

$d$ : cylinder diameter $(=19 \mathrm{~mm})$. 
$U_{e}$ : nozzle exit mean velocity.

$T_{e}$ : edgetone period.

$T_{k}$ : vortex shedding period.

$\left\langle\omega_{3}\right\rangle$ : phase averaged vorticity.

$\left\langle Q_{2}\right\rangle$ : phase averaged velocity gradient tensor.

$\langle p\rangle \quad$ : phase averaged pressure.

$x_{1}, x_{2}$ : Coordinate system with its origin at the nozzle exit.

$\tilde{u}_{1}, \widetilde{u}_{2}$ : instantaneous velocity in $x_{1}$ and $x_{2}$ directions.

$u_{1}, u_{2}$ : velocity fluctuation in $x_{1}$ and $x_{2}$ directions.

\section{Capture of coherent structures}

A large number of studies have been made on the coherent structures in a turbulence flow, and their various definitions have been adopted at every opportunity ${ }^{(9)}$. In this study, we defined the term "vortical structure" as the coherent structures with swirling motions. Coherent structures have been characterized by the use of stream line, path line, vorticity and so on ${ }^{(10)}$, and the vorticity among of them has been considered especially useful because of Galilean-invariant ${ }^{(11)}$. Also, the invariants of velocity gradient tensor were proposed by Chong \& Perry ${ }^{(12)}$ and Perry \& Chong ${ }^{(13)}$.

We briefly review these definitions in this section on the assumption that the flow is two-dimensional and incompressible. The relative velocity between an arbitrary fluid particle and anther particle which is $x_{i}$ away from there, i.e. $\dot{x}=d x_{i} / d t$, is given by using a velocity gradient tensor, $W_{i j} \equiv \partial \tilde{u}_{i} / \partial x_{j}(i, j=1,2)$.

$$
\left(\begin{array}{l}
\dot{x}_{1} \\
\dot{x}_{2}
\end{array}\right)=\left(\begin{array}{ll}
\frac{\partial \tilde{u}_{1}}{\partial x_{1}} & \frac{\partial \tilde{u}_{1}}{\partial x_{2}} \\
\frac{\partial \tilde{u}_{2}}{\partial x_{1}} & \frac{\partial \tilde{u}_{2}}{\partial x_{2}}
\end{array}\right)\left(\begin{array}{l}
x_{1} \\
x_{2}
\end{array}\right),
$$

The solution of Eq. (1) can be expressed with respect to eigenvalues $\lambda_{i}$ and eigenvectors $\zeta_{j}{ }^{i}$ of matrix $W_{i j}$. For an incompressible flow, the characteristic equation of Eq. (1) can be described as

$$
\operatorname{det}\left|\lambda \delta_{i j}-W_{i j}\right|=\lambda^{2}+Q_{2}=0
$$

where $\delta_{i j}$ is Kronecker delta and $Q_{2}$ is given by

$$
Q_{2}=\frac{\partial \tilde{u}_{1}}{\partial x_{1}} \frac{\partial \tilde{u}_{2}}{\partial x_{2}}-\frac{\partial \tilde{u}_{1}}{\partial x_{2}} \frac{\partial \tilde{u}_{2}}{\partial x_{1}}
$$

If the eigenvalues $\lambda$ have two real numbers which are distinct, the solution trajectory will show hyperbolic type, but if $\lambda$ have a conjugate complex number, the solution trajectory will show elliptic type, and the fluid particle can be considered to move with swirling motions. In general, the former has been called "saddle" while the latter has been termed "focus" to classify flow fields. The discriminate $D$ of the eigenvalues $\lambda$ equate with $-Q_{2}$ (see Eq. (2)). By taking the divergence of the Navier-Stokes equations, we find the following relationship:

$$
D=-Q_{2}=-\frac{1}{2} \nabla^{2} \widetilde{p}
$$

where $\nabla^{2} \widetilde{p}$ is laplacian for pressure. We can recognize from Eq. (4) that the local pressure fluctuations are concerned with the flow patterns i.e. saddle and focus. The identification of vortical structures based on pressure information has been proposed by Jeong \& Hussain ${ }^{(14)}$, Jeong et al. ${ }^{(15)}$ and Kida $\&$ Miura ${ }^{(16)}$.

The conditions for $\lambda$ having conjugate complex numbers are $D<0, Q_{2}>0$ and $\nabla^{2} \tilde{p}>0$. In other words, if the flow moves with swirling motions, the local minimum 
pressure will be generated within there. These relationships are grouped in Table. 2. But these distinct relationships are not necessary fixed under the three-dimensional flow fields.

Table 2 Relationship between flow pattern and the sign of $D, Q_{2}$ and $\nabla^{2} \widetilde{p}$.

\begin{tabular}{l|cccc}
\hline \hline & $\lambda$ & $D$ & $Q_{2}$ & $\nabla^{2} \tilde{p}$ \\
\hline focus & complex & $<0$ & $>0$ & $>0$ \\
saddle & real & $>0$ & $<0$ & $<0$ \\
\hline \hline
\end{tabular}

All three quantities (vorticity, $Q_{2}$ and pressure) are obviously useful information as the criterion to capture coherent structures. But in general laboratory experiments using hot wire anemometry it is very difficult to measure vorticity, and it goes without saying that it is impossible to measure $Q_{2}$ accurately. Therefore, we defined the new statistical quantities based on the phase averaging methods to detect the vortical structures. The phase average is conditioned by edgetone frequency or vortex shedding frequency, and it is represented by \langle\rangle .

$$
\begin{aligned}
& \left\langle\widetilde{\omega}_{3}\right\rangle \equiv \frac{\partial\left\langle\widetilde{u}_{2}\right\rangle}{\partial x_{1}}-\frac{\partial\left\langle\widetilde{u}_{1}\right\rangle}{\partial x_{2}}, \\
& \left\langle Q_{2}\right\rangle \equiv \frac{\partial\left\langle\widetilde{u}_{1}\right\rangle}{\partial x_{1}} \frac{\partial\left\langle\widetilde{u}_{2}\right\rangle}{\partial x_{2}}-\frac{\partial\left\langle\widetilde{u}_{1}\right\rangle}{\partial x_{2}} \frac{\partial\left\langle\widetilde{u}_{2}\right\rangle}{\partial x_{1}}, \\
& \langle\widetilde{P}\rangle \equiv P_{\infty}-P+\langle p\rangle,
\end{aligned}
$$

where $P_{\infty}$ indicates atmospheric pressure, and $P$ is time-averaged pressure. We should notice the following two points when using the phase averaged quantities for capturing the vortical structures.

(a) The vortical structures captured by the instantaneous velocity gradient tensor are the fine-scale structures whose diameter is about several times the Kolmogorov scale length. However, the structures captured by the phase averaged velocity gradient tensor are larger than them. Our analysis concentrates on quasi-two-dimensional large-scale vortical structures.

(b) Taking the divergence of the phase averaged Navier-Stokes equations, the laplacian for phase averaged pressure is obtained as

$$
\frac{1}{2} \nabla^{2}\langle\widetilde{p}\rangle=\left\langle\frac{\partial \widetilde{u}_{1}}{\partial x_{1}} \frac{\partial \widetilde{u}_{2}}{\partial x_{2}}-\frac{\partial \widetilde{u}_{1}}{\partial x_{2}} \frac{\partial \widetilde{u}_{2}}{\partial x_{1}}\right\rangle .
$$

This result means that Eq. (4) can not be satisfied in phase averaged flow fields, therefore,

$$
\left\langle Q_{2}\right\rangle \neq \frac{1}{2} \nabla^{2}\langle\widetilde{p}\rangle .
$$

In this study, three regions were used as criteria to capture the coherent structures: (i) the region where the phase-averaged vorticity $\left\langle\widetilde{\omega}_{3}\right\rangle$ indicates a large value, (ii) the local minimum region of phase-averaged pressure $\langle\widetilde{p}\rangle$, and (iii) the region where the phase-averaged velocity gradient tensor $\left\langle Q_{2}\right\rangle$ indicates a large positive value. These three regions are independent of each other. As we will see later in the wake experiment, if the region (ii) coincides with the region (iii), we regard them as coherent structures moving with swirling motions, and call them "vortical structures". 


\section{Results and discussion}

The results of capturing vortical structures in a low-Reynolds number wake of a cylinder and high-Reynolds number edgetone flow are shown in this section. The criteria to capture them are (i), (ii) and (iii), as respectively defined in section 3. Although the quantity $Q_{2}$ is equivalent to the pressure fluctuation through Eq. (4), this relationship is not satisfied in phase averaged flow fields, so it is not clear how accurately $\left\langle Q_{2}\right\rangle$ can capture vortical structures. Therefore, we first try to educe the specific vortical structures in Karman vortex sheet, and then discuss capturing the vortical structures in edgetone flow on the basis of the results.

\subsection{Pressure measurements in a wake of cylinder}

The mean pressure is related with the following Reynolds equations in $x_{2}$ direction,

$$
\overline{u_{1}} \frac{\partial \overline{u_{2}}}{\partial x_{1}}+\overline{u_{2}} \frac{\partial \overline{u_{2}}}{\partial x_{2}}=-\frac{1}{\rho} \frac{\partial P}{\partial x_{2}}+v \frac{\partial^{2} \overline{u_{2}}}{\partial x_{1}{ }^{2}}+v \frac{\partial^{2} \overline{u_{2}}}{\partial x_{2}{ }^{2}}-\frac{\partial \overline{u_{1} u_{2}}}{\partial x_{1}}-\frac{\partial \overline{u_{2}{ }^{2}}}{\partial x_{2}} .
$$

Equation (10) is simplified near the center line as follows:

$$
\frac{1}{\rho} \frac{\partial P}{\partial x_{2}}+\frac{\partial \overline{u_{2}^{2}}}{\partial x_{2}}=0
$$

And the integration from the center line to a point far from there is:

$$
\overline{u_{2}^{2}}=\left(P_{\infty}-P\right) / \rho \text {. }
$$

It is apparent that the mean static pressure $P$ is estimated by measuring the vertical turbulent intensity $\overline{u_{2}^{2}}$.

The distribution of $\rho \overline{u_{2}{ }^{2}}$ and $\left(P_{\infty}-P\right) / \rho$ at $x_{1} / d=5$ are shown in Fig. 4. Both values have maximum at center line, and good agreement is shown at around $-0.5 \leq x_{2} / d \leq 0.5$. Therefore, we can recognize that the accurate mean static pressure measurements are achieved. The difference between both values increases as the profiles approach the edge. We assume that this disagreement results from the fact that the boundary layer assumption does not hold there.

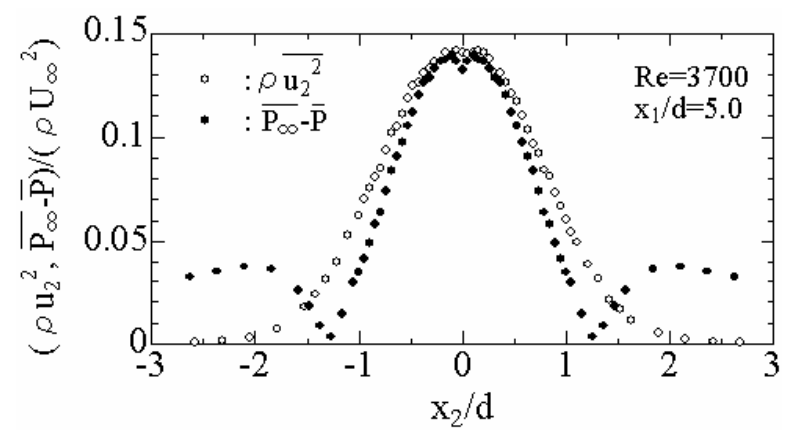

Fig. 4 Comparison between measured static pressure profile and velocity fluctuation intensity $\overline{\rho u_{2}{ }^{2}}$. Note that two quantities should agree [Eq. (12)] when the boundary layer assumption holds.

The root mean square value of pressure fluctuations $\left(\overline{p^{\prime}}\right)$ and the pressure-velocity correlations $\left(\overline{p u_{1}}, \overline{p u_{2}}\right)$ are shown in Fig. 5(a). And Kobashi et al's results of initial condition $\operatorname{Re} \sim 9000$ at $x_{1} / d=42$ are shown in Fig.5 (b) for comparison ${ }^{(17)}$. The quantitative comparison is difficult between the present measurements and Kobashi et al's results because of different experimental conditions. For the moment, let us look closely at a comparison with the distribution profiles. 


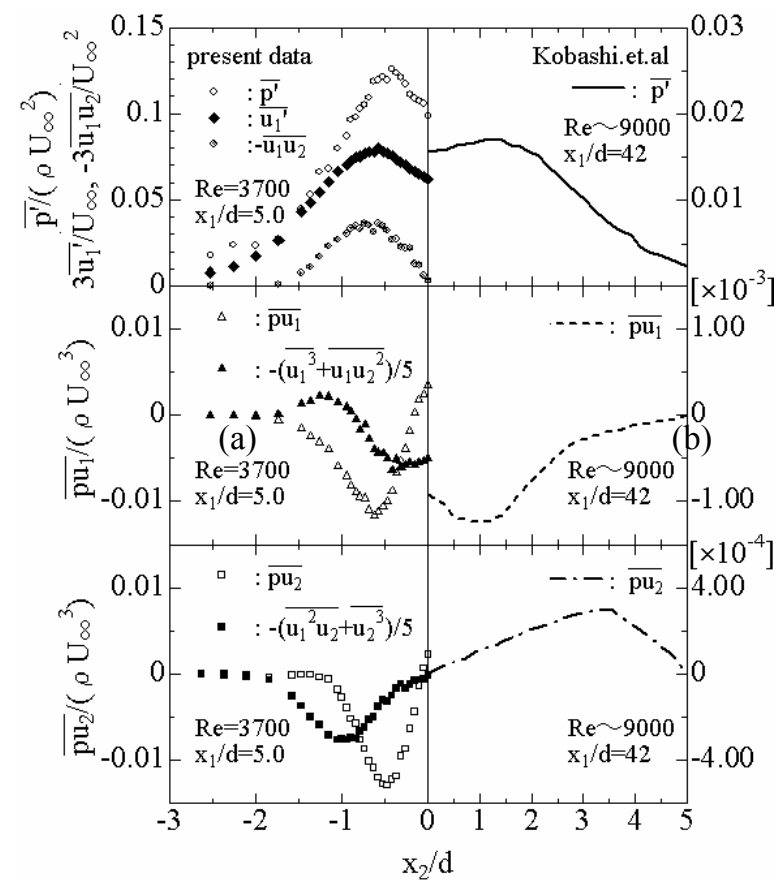

Fig. 5 (a) Cross-sectional distributions of $\overline{p^{\prime}}, \overline{p u_{1}}, \overline{p u_{2}} . \bigcirc, \triangle$, and $\square$ : present measurements, $\boldsymbol{\Delta}$ and $\boldsymbol{\square}$ : Lumley model calculation [Eq. (13)] based on measured velocities at $x_{1} / d=5$. (b) Kobashi et al's results of $\mathrm{Re}=9000$ at $x_{1} / d=42^{(17)}$.

The distributions $\overline{p^{\prime}}$ and $\overline{p u_{1}}$ show symmetry for the center line, and distributions $\overline{p u_{2}}$ show symmetry for wake center point, therefore all distributions are shown in $x_{2} / d \leq 0$ for convenience. Few studies on the statistical quantities of pressure fluctuations have been reported so far, making it difficult to quantitatively evaluate the present measurements. But several derived features are as follows.

The present $\overline{p^{\prime}}$ distribution has a minimum at the center line and a maximum at $\left|x_{2} / d\right|=0.5$. This maximum position is also closer to the center line than the $u_{1}$ root mean square value $\left(u_{1}{ }^{\prime}\right)$ and Reynolds stress $-\bar{u}_{1} u_{2}$. A similar tendency was obtained qualitatively in Kobashi et al's results. The distribution of pressure-velocity correlation $\overline{p u_{1}}$ shows symmetry for the center line, which indicates a positive correlation on the center line and a negative correlation around the verge of the wake. The negative correlation has minimum intensity at $\left|x_{2} / d\right|=0.5$. The distribution of $\overline{p u_{2}}$ shows symmetry with the wake center point, indicates a negative correlation in $x_{2} / d<0$ and a positive correlation in $x_{2} / d>0$. These correlations reach down to $\left|x_{2} / d\right|<1$ but the positive (or negative) correlation region is narrower than that of $\overline{p u_{1}}$. Also, in the case of Kobashi et al's measurements, the peaks of $\overline{p u_{1}}$ and $\overline{p u_{2}}$ locate further from the center line than our results.

Lumley proposed the following model to solve the Reynolds stress equation:

$$
-\overline{p u_{i}} / \rho=\overline{u_{i} u_{j} u_{j}} / 5
$$

The above equation was derived by applying the homogeneous condition to the slow source terms of $\nabla^{2} \widetilde{p}^{(18,19)}$. The pressure-velocity correlations are calculated by Eq. (13) but $j=1,2$ are shown in Fig. 5. Quantitative agreement between theory and experiments will be difficult because no $u_{3}$ measurement is performed in this study. However, Aronson \& Lofdahl ${ }^{(20)}$ reported the similar distribution in case of $j=3$. Comparing the present results with Lumley's model, we find they are close with each other around the centerline $\left(x_{2} / d=0\right)$. But there is a great difference in the minimum location. It is supposed this region does not hold the homogeneous turbulence condition to satisfy Eq. (13). This 
disagreement also includes measurement errors for the velocity-pressure simultaneous measurements ${ }^{(8)}$. The pressure probe and hot-wire probe can not be measured at the same position, so the gap in the measurement positions and each probe interfering with flow fields will affect measurements of the correlation coefficient. This effect becomes more pronounced as the Reynolds number increases or finer-scale structures appear in the flow.
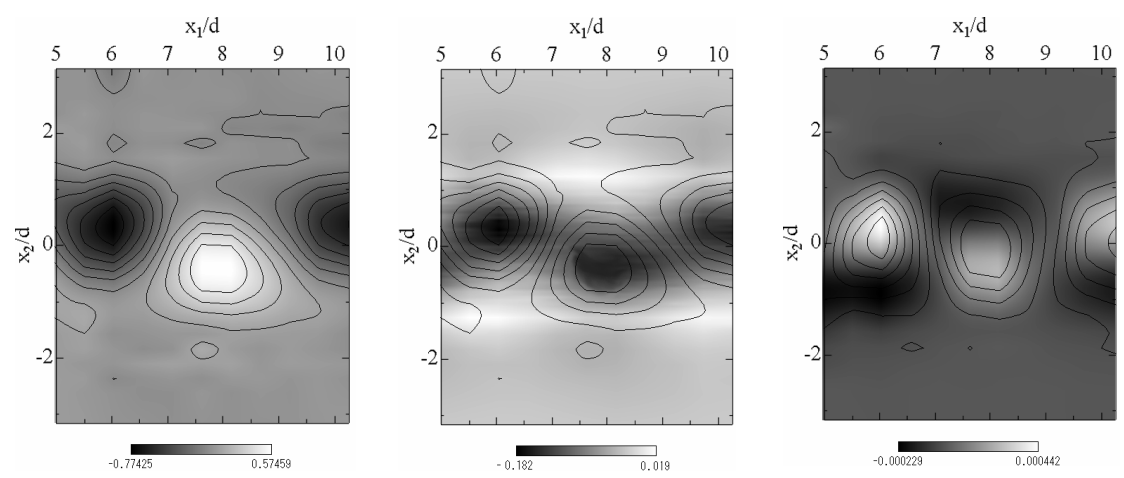

Fig. 6 Contour plots of phase-averaged quantities at phase angle of $T_{k} / 8$.(a) phase-averaged vorticity $\left\langle\widetilde{\omega}_{3}\right\rangle$, (b) phase-averaged pressure $\langle\widetilde{p}\rangle$, (c) phase-averaged velocity gradient tensor $\left\langle Q_{2}\right\rangle$. Overlaid are phase averaged vorticity contour lines as indicated in Fig. 6(a).

\subsection{Coherent structures in low-Reynolds number wake of cylinder}

Figure 6(a) shows the phase averaged vorticity $\left\langle\widetilde{\omega}_{3}\right\rangle /\left(U_{\infty} / d\right)$ contour plot derived from the wake of cylinder at $T_{k} / 8\left(T_{k}\right.$ : vortex shedding period). Brighter position indicates higher intensities. The distribution holds the concentrated positive vorticity in the fields below the center line $\left(x_{2} / d=0.0\right)$ and negative vorticity region appears in upper fields. The well-known asymmetrically-arranged coherent structures are apparent. The phase averaged pressure $\langle\widetilde{p}\rangle$ contour plot is shown in Fig. 6(b) at the same phase angle as in Fig. 6(a). The phase averaged vorticity contour lines shown in Fig. 6(a) are overlaid for comparison. The phase averaged pressure keeps negative pressure almost entirely around the test section, which is lower than atmospheric pressure as indicated by Eq. (12). Note that the local minimum pressure region corresponds with concentrated vorticity region shown by contour lines ${ }^{(21)}$. The contour plot of $\left\langle Q_{2}\right\rangle$ is shown in Fig. 6(c), where the phase averaged vorticity contour lines are overlaid as in Fig. 6(b). The $\left\langle Q_{2}\right\rangle$ has a positive value in the center of the concentrated vorticity region, and the focus region corresponds with the coherent structures captured by $\left\langle\widetilde{\omega}_{3}\right\rangle$. The middle location between adjoining structures indicates the saddle region because $\left\langle Q_{2}\right\rangle$ shows a negative value in there. Note that the focus and saddle regions correspond with the low and high phase averaged pressure region, respectively, in Fig. 6(b). This relationship between $\langle\widetilde{p}\rangle$ and $\left\langle Q_{2}\right\rangle$ reflects good agreement as shown in Table 2. For the reasons mentioned above, it is obvious that the phase-averaged velocity gradient tensor works well to capture the coherent structures with swirling motions (i.e. vortical structures).

\subsection{Coherent structures in high-Reynolds number edgetone flow}

Figure 7 (a) and (b) show the phase averaged vorticity contour plots in the edgetone experiments at a fixed phase $7 T_{E} / 8$ and $8 T_{E} / 8$ ( $T_{E}$ : edgetone period). The brighter position indicates the higher intensities as shown in Fig. 6 . The circle plots $(\circ)$ shown in the figure indicate the location where streamwise phase averaged velocity $\left\langle\widetilde{u}_{1}\right\rangle$ has the maximum value in the $x_{2}$ direction. Other phase results can not be shown for lack of space. The circle plots vibrate up and down as the edgetone phase angle varies, which correspond 
to the periodic vibration of jet axis. In the impinging region $\left(x_{1} / b_{e} \leq 20\right)$, the phase averaged vorticity indicates the maximum magnitude at the jet shear layer near the nozzle exit, and the monotonic decay toward the leading edge of the flat plate. But in the impinging region, we can not find the area where the vorticity concentrates and indicates the coherent structures. However, the concentrated vorticity region appears in the wall region at $x_{1} / b_{e} \approx 30$, and is then transported along the flat plate (see Fig. 7(a), (b)). The phase averaged pressure $\langle\tilde{p}\rangle$ at the same phase angle as in Fig. 7 is shown in Fig. 8(a), (b). The phase averaged vorticity in Fig. 7 is overlaid by contour lines for comparison. We measured the pressure fluctuations only for field below the center line due to pressure probe geometry. The negative pressure region appears locally in the impinging region at $x_{1} / b_{e} \approx 15$, which implies the existence of coherent structures. In the wall region at $x_{1} / b_{e} \approx 30$, the concentrated phase averaged vorticity region is captured in the Fig. 7 overlay with the local minimum pressure region. Thus, $\left\langle\widetilde{\omega}_{3}\right\rangle$ does not correspond with $\langle\widetilde{p}\rangle$ in the impinging region, but they are in good agreement with each other in wall region.

The contour plots of $\left\langle Q_{2}\right\rangle$ are shown in Fig. 9(a) and (b), and the phase averaged vorticity contour lines are overlaid as in Fig. 8. The clear positive-concentrated region (i.e. focus region) exists locally in the impinging region at $x_{1} / b_{e} \approx 15$ in Fig. 9(a). This captured focus region corresponds with the local minimum pressure region in Fig. 8(a). The trajectory of the focus regions captured in each phase angle indicates that coherent structures are formed at the impinging region and are then transported toward the wall region. Also, the focus regions corresponded well with the local minimum pressure region at all phase angles (figures are not shown). In short, the distributions of $\langle\widetilde{p}\rangle$ and $\left\langle Q_{2}\right\rangle$ are in good agreement in the impinging region and wall region. Therefore, it seems that the vortical structures defined in this study exist in both regions.

As mentioned above, the distribution $\left\langle\widetilde{\omega}_{3}\right\rangle$ is far different from the $\langle\widetilde{p}\rangle$ and $\left\langle Q_{2}\right\rangle$ distributions in the impinging region. We shall discuss the reason in detail using three-component analysis, which is denoted as follows:

$$
\widetilde{u}_{i}=\bar{u}_{i}+u_{i}=\bar{u}_{i}+u_{i \theta}+u_{i s},
$$

where $u_{i \theta}$ indicates the periodic component of fluctuation velocity $u_{i}$, and the rest of $u_{i}$ is expressed as $u_{i s}$, called the non-periodic component in this study ${ }^{(7)}$. By applying the phase averaged operator to Eq. (14), the non-periodic component $u_{i s}$ will disappear:

$$
\left\langle\tilde{u}_{i}\right\rangle=\bar{u}_{i}+\left\langle u_{i \theta}\right\rangle
$$

Moreover, if the phase averaged velocity can be decomposed into the contribution from vortical structures $\left\langle u_{i c}\right\rangle$ and the other components $\left\langle u_{i b}\right\rangle$ exactly, the following expression will be produced instead of Eq. (15).

$$
\left\langle\tilde{u}_{i}\right\rangle=\left\langle u_{i b}\right\rangle+\left\langle u_{i c}\right\rangle
$$

$\left\langle u_{i b}\right\rangle$ includes time-averaged velocity and so on, which is called background velocity in what follows. As we impose the condition of Eq. (16) upon the phase averaged vorticity $\left\langle\widetilde{\omega}_{3}\right\rangle$, it was decomposed as follows:

$$
\begin{gathered}
\left\langle\widetilde{\omega}_{3}\right\rangle=\left\langle\omega_{3 b}\right\rangle+\left\langle\omega_{3 c}\right\rangle . \\
\left\langle\omega_{3 b}\right\rangle=\frac{\partial\left\langle u_{2 b}\right\rangle}{\partial x_{1}}-\frac{\partial\left\langle u_{1 b}\right\rangle}{\partial x_{2}} . \\
\left\langle\omega_{3 c}\right\rangle=\frac{\partial\left\langle u_{2 c}\right\rangle}{\partial x_{1}}-\frac{\partial\left\langle u_{1 c}\right\rangle}{\partial x_{2}} .
\end{gathered}
$$



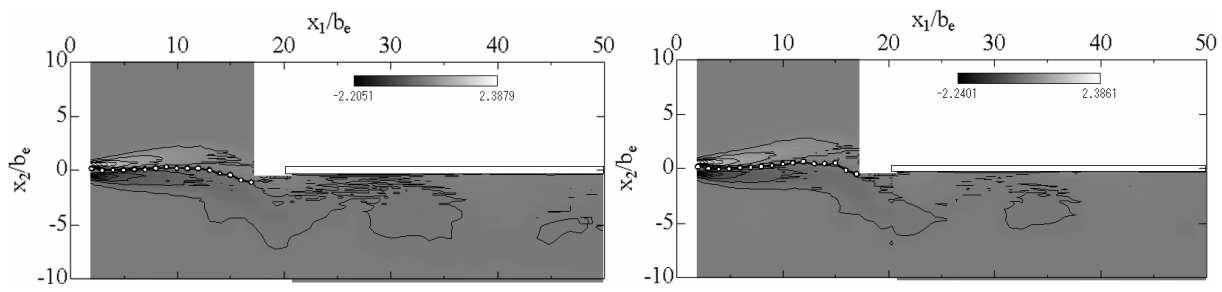

Fig. 7 Contour plot of phase-averaged vorticity, $\left\langle\widetilde{\omega}_{3}\right\rangle$ at fixed phase angles, (a) $7 T_{e} / 8$, (b) $8 T_{e} / 8$.
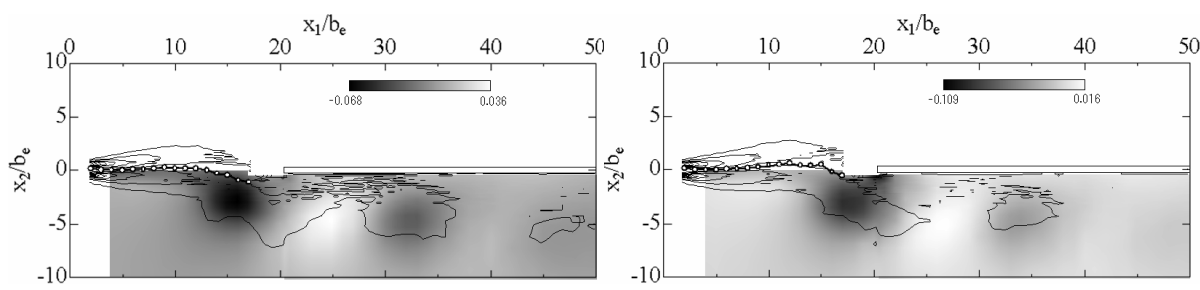

Fig. 8 Contour plot of phase-averaged pressure, $\langle\tilde{p}\rangle$ at fixed phase angles, (a) $7 T_{e} / 8$, (b) $8 T_{e} / 8$.
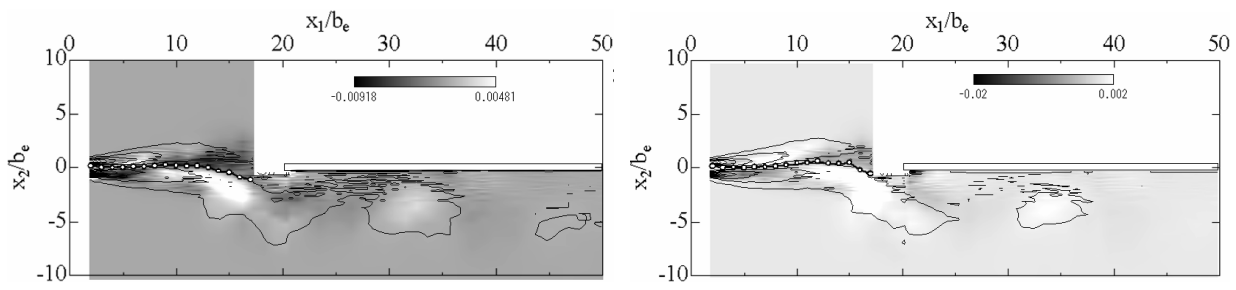

Fig. 9 Contour plot of phase-averaged velocity gradient tensor, $\left\langle Q_{2}\right\rangle$ at fixed phase angles, (a) $7 T_{e} / 8$, (b) $8 T_{e} / 8$.

Where $\left\langle\omega_{3 b}\right\rangle$ shows the vorticity contributed from the background velocity, and vorticity $\left\langle\omega_{3 c}\right\rangle$ includes only the contribution from vortical structures. In the wake of our circular cylinder experiment at low Reynolds number, $\left\langle\widetilde{\omega}_{3}\right\rangle$ worked well to capture the vortical structures. This is by the reason that $\left\langle\omega_{3 c}\right\rangle$ is much larger than $\left\langle\omega_{3 b}\right\rangle$ $\left(\left\langle\omega_{3 c}\right\rangle>\left\langle\omega_{3 b}\right\rangle\right)$. On the other hand, the reason why $\left\langle\widetilde{\omega}_{3}\right\rangle$ could not capture well the vortical structures in high-Reynolds number edgetone experiments for the impinging region is that the contribution from $\left\langle\omega_{3 b}\right\rangle$ to $\left\langle\widetilde{\omega}_{3}\right\rangle$ is much larger than that of the wake. Of course, it is debatable whether or not these differences were caused by the flow field differences. But in general, as the Reynolds number becomes higher, the contribution from $\left\langle\omega_{3 b}\right\rangle$ to $\left\langle\widetilde{\omega}_{3}\right\rangle$ is larger than $\left\langle\omega_{3 c}\right\rangle$. Thus, the vortcity is misleading when the phase averaged technique is used.

The exact decomposition of $\left\langle\widetilde{u}_{i}\right\rangle$ into $\left\langle u_{i b}\right\rangle$ and $\left\langle u_{i c}\right\rangle$ has never been reported as far as we know. But we once tried to extract the coherent structures by assuming that the time-averaged velocity $\overline{u_{i}}$ and phase averaged periodic components $\left\langle u_{i \theta}\right\rangle$ correspond with $\left\langle u_{i b}\right\rangle$ and $\left\langle u_{i c}\right\rangle$, respectively, and defined $\left\langle\omega_{3 \theta}\right\rangle$ in the previous work ${ }^{(7)}$ as

$$
\left\langle\omega_{3 \theta}\right\rangle=\frac{\partial\left\langle u_{2 \theta}\right\rangle}{\partial x_{1}}-\frac{\partial\left\langle u_{1 \theta}\right\rangle}{\partial x_{2}} .
$$

However, the structures based on $\left\langle\omega_{3 \theta}\right\rangle$ passed along the center line $\left(x_{2} / b_{e}=0.0\right)$, and it was apparent that they were different from the structures captured by $\langle\widetilde{p}\rangle$. The reason for this disagreement is that the time-averaged velocity would include some parts of velocity components induced by vortical structures $\left\langle u_{i c}\right\rangle$. Hence, it seems to be surplus that 
time-averaged velocity is subtracted as the background velocity from $\left\langle\widetilde{\omega}_{3}\right\rangle$.

Applying the three-component analysis to Eq. (4), then using phase averaging, the Poisson equation for the phase-averaged pressure field is obtained as

$$
\frac{1}{2} \nabla^{2}\langle\widetilde{p}\rangle=-\frac{1}{2} \frac{\partial\left\langle\tilde{u}_{i}\right\rangle}{\partial x_{j}} \frac{\partial\left\langle\tilde{u}_{j}\right\rangle}{\partial x_{i}}-\frac{1}{2} \frac{\partial^{2}\left\langle u_{i s} u_{j s}\right\rangle}{\partial x_{i} \partial x_{j}} .
$$

It should be noted that the first term on the right-hand side of Eq. (21) equals $\left\langle Q_{2}\right\rangle$. In the wake of circular cylinder experiments and edgetone experiments, the positive regions of $\left\langle Q_{2}\right\rangle$ corresponded well with the negative pressure region $\langle\widetilde{p}\rangle$. This result also shows that the first term on the right-hand side of Eq. (21) will be pronounced in both flow fields. Thus, the following relationship will be satisfied there:

$$
\frac{1}{2} \nabla^{2}\langle\widetilde{p}\rangle \approx-\frac{1}{2} \frac{\partial\left\langle\widetilde{u}_{i}\right\rangle}{\partial x_{j}} \frac{\partial\left\langle\tilde{u}_{j}\right\rangle}{\partial x_{i}}=\left\langle Q_{2}\right\rangle .
$$

We may conclude from the foregoing that, in the case of flow fields which can be approximated as two-dimensional, the most accurate method to capture the vortical structures among the three definitions is $\langle\widetilde{p}\rangle$, and if the phase-averaged pressure field is governed by Eq. (22), the quantity $\left\langle Q_{2}\right\rangle$ will work well. Moreover, when the background velocity components $\left\langle u_{i b}\right\rangle$ are much smaller than $\left\langle u_{i c}\right\rangle$, the phase averaged vorticity $\left\langle\widetilde{\omega}_{3}\right\rangle$ will capture well the vortical structures.

\subsection{Convection speeds of coherent structures}

As we have mentioned in the preceding chapter, the coherent structures captured by $\left\langle Q_{2}\right\rangle$ and $\langle\tilde{p}\rangle$ corresponded well with each other in the high-Reynolds number edgtone flow as well as the low-Reynolds number wake of a cylinder. This result leads to the idea that the edgetone vortices captured so far by other researchers are vortical structures.

When the local maximum points of $\left\langle Q_{2}\right\rangle$ are defined as the center of the vortical structures, the convection speed of vortical structures is evaluated by calculating the time variation of the center location. The center of the vortical structure in terms of the $x_{1}$ coordinate is shown in Fig. 10. The dot-dashed line indicates the location of the leading edge of the flat plate. We divide this distribution into the impinging region and wall region, then calculate the convection speed $C_{x}$ in $x_{1}$-direction from the distribution slopes for each region. The results show that $C_{x}=8.0 \mathrm{~m} / \mathrm{s}$ in impinging region and $C_{x}=6.5 \mathrm{~m} / \mathrm{s}$ in wall region. In addition, we estimate the $x_{2}$-direction convection speed to be $C_{y} \approx 0.6 \mathrm{~m} / \mathrm{s}$, which is much smaller than $C_{x}$. The flow fields in which the convection speeds are subtracted from the phase averaged velocity $\left(\left\langle\tilde{u}_{1}\right\rangle-C_{x},\left\langle\widetilde{u}_{2}\right\rangle-C_{y}\right)$ are shown in Figs. 11(a) and (b). These phases correspond with $7 T_{E} / 8$ and $8 T_{E} / 8$, respectively. As mentioned above, $C_{y}$ is much smaller than $C_{x}$, so we can recognize Figs. 11(a) and (b) are the velocity-vector fields viewed from a reference frame moving with the vortical structure. The contour plots of $\langle\tilde{p}\rangle$ captured in Figs. 8(a) and (b) are overlaid for comparison. The velocity vectors show the swirling motion having their center in the local minimum region of $\langle\tilde{p}\rangle$. This result leads support to the notion that the coherent structures in Fig. 8 and 9 involve a swirling motion.

We have to focus attention on the relationship between the convection speed and edgetone phenomenon. The mechanism of edge tone has been explained by feedback loop due to the sound noise induced by vortical structure impingement as follows ${ }^{(1)}$. Large-scale vortical structures impinging on the leading edge cause the local pressure sources around there. The created pressure will propagate in the radial direction, reach the nozzle exit, and 
the vorticity will be generated there because of shear instability. This induced vorticity increases downstream due to Kelvin-Helmholtz instability and impinges on the object again, so that the feedback loop closes.

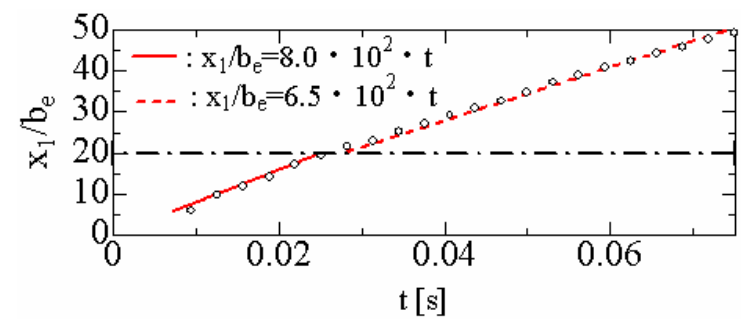

Fig. 10 Location of maximum point of $\left\langle Q_{2}\right\rangle$ in downstream direction as a function of time.

Based on this pressure-feedback hypothesis, Powell reported that the following relationship would be satisfied ${ }^{(1,22)}$.

$$
\frac{\ell}{u_{p}}+\frac{\ell}{a_{0}}=n / f_{e},
$$

where $\ell$ is the distance from pressure sources to jet sensitive region, $u_{p}$ and $a_{0}$ indicate the convection speed of vortical structures and sound speed, respectively. Also $n$ and $f_{e}$ show oscillation stage and edgetone frequency, therefore $n / f_{e}$ correspond to edgetone period. The present oscillation stage is $n=1$ because of the jet center-line oscillation showed in Fig. 7. Assuming that $\ell$ also corresponds to the distance from nozzle exit to leading edge $\left(=20 b_{e}\right)$, we can obtain the convection speed $u_{p} \approx 8.0 \mathrm{~m} / \mathrm{s}$ in the present condition by substituting $f_{e}=40$ and $a_{0}=344 \mathrm{~m} / \mathrm{s}$ into Eq. (16). Note that this convection speed is also agreement with voritical structure's convection speed $C_{x}$ in impinging region calculated in Fig. 10. Therefore, we recognize that the vortical structures captured by $\left\langle Q_{2}\right\rangle$ and $\langle\widetilde{p}\rangle$ will be the vortex in the feedback mechanism, and its convective speed will determine the edgetone frequency.
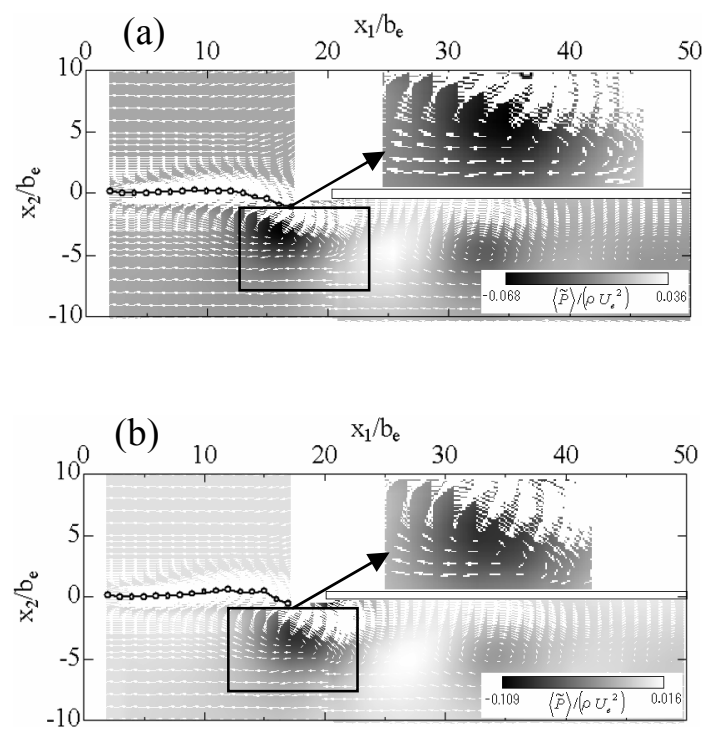

Fig. 11 Velocity field viewed from reference frame moving downstream at speed $C_{x}$. Phase is fixed at (a) $7 T_{e} / 8$, (b) $8 T_{e} / 8$. Overlaid is a contour plot of phase averaged pressure indicated in Fig. 8 (a), (b). 


\section{Summary and conclusions}

Coherent structures in the high-Reynolds number edgetone oscillations and low-Reynolds number wake of a cylinder are captured by three statistical quantities; (i) the phase-averaged vorticity $\left\langle\widetilde{\omega}_{3}\right\rangle$, (ii) the phase-averaged pressure $\langle\widetilde{p}\rangle$, and (iii) the phase-averaged velocity gradient tensor $\left\langle Q_{2}\right\rangle$. Defining the term vortical structure as the coherent structures with swirling motions, we have discussed the existence of vortical structures in high-Reynolds number edgetone flow from comparison of the coherent structures captured in the low-Reynolds number wake. The results might be summarized as follows.

(1) In the low-Reynolds number wake, the coherent structures captured by the above three quantities are in good agreement with one another.

(2) In the high-Reynolds number edgetone flow, the coherent structures captured by $\langle\widetilde{p}\rangle$ correspond well with $\left\langle Q_{2}\right\rangle$ in the impinging region, so the vortical structures seem to be included as well as the wall region. However, the coherent structures based on $\left\langle\widetilde{\omega}_{3}\right\rangle$ disagree with them because the background velocity gradients are larger than that of the low-Reynolds number wake, and $\left\langle\widetilde{\omega}_{3}\right\rangle$ is much affected by them.

(3) Convection speeds are estimated by the location where $\left\langle Q_{2}\right\rangle$ indicates the local maximum value. The result shows that the convection speed is about $8.0 \mathrm{~m} / \mathrm{s}$ in impinging region, which satisfies the relation of the Powell feedback hypothesis. This result supports that the coherent structures captured by $\langle\widetilde{p}\rangle$ or $\left\langle Q_{2}\right\rangle$ contribute to the feedback mechanism. By subtracting the convection speeds from the phase averaged velocity, it is found that the coherent structures with swirling motions are included in high-Reynolds number edgetone flow. This result implies that edgetone vortices are vortical structures.

\section{References}

(1)Powell, A., On the edgetone, J. Acous. Soc. America., Vol. 33-4(1961), pp. 395-409.

(2)Rockwell, D., Oscillations of impining shear layers, AIAA Journal, Vol.21(1983), pp.645-664.

(3)Ziada, S., Feedback control of globally unstable flows: impinging shear flows, Journal of Fluids and Structure, vol.9, (1995), pp.907-923.

(4)Micheal, L., Rockwell, D., Self-excited jet: upstream modulation and multiple frequencies, Journal of Fluid Mech., Vol. 147(1983), pp. 333-352.

(5)Popiel. C. O., Trass, O., Visualization of a free and impinging round jet, Experimental Thermal and Fluid Science, Vol.4(1991), pp.253-264.

(6)Kawai, Y., Tsuji, Y., Kukita, Y., Characteristics of coherent structures in a jet indicating edgetone oscillations $1^{\text {st }}$ report, Transaction of the JSME, Series B, 70-692(2004-4), pp. 935-942.

(7)Kawai, Y., Tsuji, Y., Kukita, Y., Characteristics of coherent structures in a jet indicating edgetone oscillations $2^{\text {nd }}$ report, Transaction of the JSME, Series B, 72-716(2006-4), pp. 915-923.

(8)Tsuji, Y., Fransson, J.H.M., Alfredson,P.H., and Johansson A.V., Pressure statistics and their scaling in high-Reynolds number turbulent boundary layers, JFM., Vol.585, (2007), pp.1-40.

(9)Cantwell, B. J., Coles, D., An experimental study of entrainment and transport in the turbulent near wake of a circular cylinder, Journal of Fluid Mech., Vol. 136(1983), pp. 321-374.

(10)Hussain, A. K. M. F., Zaman, K. B. M. Q., Vortex pairing in a circular jet under controlled excitation, Journal of Fluid Mech., Vol. 101(1980), pp. 493-544.

(11)Hussain, A. K. M. F., Coherent structures - reality and myth, Phys. Fluids., Vol. 26-10(1983), pp. 2816-2850.

(12)Chong, M. S., Perry, A. E., Cantwell, B., A general classification of theree-dimensional flow fields, Phys. Fluids A, Vol.2(1990), pp. 765-777.

(13)Perry, A. E., Chong, M. S., A description of eddying motions and flow patterns using critical-point concepts, Ann. Rev. Fluid Mech., Vol. 19(1987), pp. 125-155. 
(14)Jeong, J., Hussain, F., On the identification of vortex, Journal of Fluid Mech., Vol. 285(1995), pp. 69-94.

(15)Jeong, J., Hussain, F., Schoppa, W., Kim, J., Coherent structure near the wall in a turbulent channel flow, Journal of Fluid Mech., Vol. 332(1997), pp. 185-214.

(16)Kida, S., Miura, H., Identification and analysis of vortical structure, Eur. J. Mech. B/Fluids, 17(1998), pp. 471-488.

(17)Kobashi, Y., Measurement of pressure fluctuation in the wake of cylinder, J. Physical Soc. Japan., Vol. 12(1957), pp. 533-543.

(18)Lummley, J. L., Computational modeling of turbulent flows, Advances in Applied Mechanics., Vol.18(1978), pp. 123-176.

(19)Naka, Y., Omori, T., Obi, S., Masuda, S., Experimental study on velocity-pressure correlation in turbulent mixing layer out of equilibrium state, TSFP4. Vol. 1(2005), pp. 1125-1130.

(20)Aronson, D., Lofdahl, L., The plane wake of a cylinder: An estimate of the pressure-strain rate tensor, Phys. Fluids, Vol. 6(1994), pp. 2716-2721.

(21)Shirahama, Y., Toyoda, K., Development of the probe to measure static-pressure fluctuations, Transaction of the JSME, Series B, 59-567(1993-11), pp. 3381-3387.

(22)Kiya, M., Motizuki, O., Park, J. W. Shirahama, Y., Experiments on response of impinging jet and spanwise coherence of the jet, J. Sound and Vibration., Vol. 188(1995), pp. 3-16.

(23)Narashimuha, R., Prasad, S. N., Leading edge shape for flat plate boundary layer studies, Experiments in Fluids, vol.17(1994), pp.358-374. 